\title{
Exploiting the Physiochemical Interactions between Single-Walled Carbon Nanotubes and Hydrogel Microspheres to Afford Chirally Pure Nanotubes
}

\author{
Brennan P. Watts, ${ }^{\dagger}$ Cameron H. Barbee, ${ }^{\dagger}$ and Kevin Tvrdy ${ }^{\dagger *}$ \\ ${ }^{\dagger}$ Department of Chemistry \& Biochemistry; University of Colorado Colorado Springs; Colorado Springs, \\ Colorado \\ *ktvrdy@uccs.edu
}

\section{Table of Contents}

Section

$\underline{\text { Page }}$

S1. Detailed Materials and Methods

S-1

S2. Spectroscopic deconvolution of SWNT absorbance spectra S-5

S3. Geometric model of SWNT interaction with curved surface of hydrogel microsphere

S4. Comparison between column-based and centrifuge-based methods to achieve gel/SWNT phase separation within SWNT purification scheme 


\section{S.1 Detailed Materials and Methods}

\section{S1.1 Materials and Equipment}

- Sephacryl S-200 High Resolution (Sephacryl) obtained from GE Healthcare Bio-Sciences AB, (Uppsala, Sweden) lot \# $10240334-750 \mathrm{~mL}$ preparatory grade.

- Sodium dodecyl sulfate $\geq 98.5 \%$ (SDS) obtained from Sigma Life Science, (St. Louis, MO) lot\# SLBM8498V

- Carbon nanotubes, single walled enriched in $(6,5)$ chirality $(\geq 95 \%$ carbon basis $[\geq 95 \%$ as carbon nanotubes]) obtained from Sigma Aldrich (CAS 308068-56-6, mfr. no. Signis ${ }^{\circledR}$ SG65i )

- Rod shaped stir bar $7.5 \mathrm{~cm} \times 1 \mathrm{~cm}$ employed for the fractionation of Sephacryl at high stir speeds within Pyrex 500mL Erlenmeyer flask No. 4980, stopper No. 7, stir speed of 150 RPM upon Isotemp ${ }^{\circledR}$ digital stirring hot plate: ceramic (catologue \# 11-300-49SHP)

- 120v Digital tube revolver obtained from thermo scientific (catalogue \#88881001) and employed during the centrifugation/decanting SWNT/gel interaction procedure

- $120 \mathrm{~V} 60 \mathrm{~Hz}$ Sorvall ST 16 Centrifuge obtained from ThermoFisher Scientific (Catalogue \#75004241) and employed for general purpose centrifugation

- Sorvall MX 120 Plus Micro-Ultracentrifuge obtained from ThermoFisher Scientific and employed both for treatment and processing of SWNT as well as Sephacryl with S50-A fixed angle rotor

- HP Agilent 8453 G1103A spectrometer obtained from Agilent Technologies and employed for spectroscopic analysis of SWNT products post-interaction

- Leica DM750 M Binocular Materials Microscope fitted with Leica ICC50 HD Microscope Camera employed for sizing and microscopic analysis of hydrogel samples

- Karter Scientific Labware Manufacturing 72pc glass microscope slides and 100 cover slip set, employed for microscopic analysis of samples.

\section{S1.2 Methods}

\section{S1.2.1 Size segregation of hydrogel microspheres}

The commercially available hydrogel Sephacryl S-200 (Sephacryl) consists of hydrogel beads with diameter distribution of 25 to $75 \mu \mathrm{m}$. To support the hypothesis that Sephacryl-based SWNT purification schemes rely on surface interactions, rather than interior sampling (as is typically the case in size exclusion chromatography), it was necessary to generate samples of Sephacryl with varied surface area to material mass ratio. Samples were prepared by loading and homogenizing $1.5 \mathrm{~mL}$ of Sephacryl and $13.5 \mathrm{~mL}$ of aqueous $70 \mathrm{mM}$ SDS solution into $15 \mathrm{~mL}$ falcon tubes and allowing aliquots to settle under gravity. This process afforded a vertical gradient of bead diameter within the sample container - larger beads were found to exist in higher proportions near the bottom of the solution and smaller proportions near the top of the solution. Subsequent decanting of the settled beads and segregation into vertical layers resulted in aliquots with varied particle diameter distributions. Decanting was afforded by gentle agitation of the upper layer of settled beads, followed by gentle swirling of the solution and removal of the uppermost layer.

Because the efficiency of bead segregation is a function of the solvent to settled hydrogel ratio, segregation experiments were conducted in both solvent dominant (9:1 solvent:gel by volume) and gel dominant (1:9 solvent:gel by volume) environments. Segregation of both samples into a total of five layers each, coupled with microscopic analysis, revealed a maximum difference in mean diameter between segregated and native samples $(\Delta \mathrm{d})$ of $6.57 \mu \mathrm{m}$ for gel dominant environments and $11.98 \mu \mathrm{m}$ for solvent 
dominant environments. The same procedure was also conducted via centrifugation in place of gravitational settling, but ultimately resulted in a smaller value of $\Delta \mathrm{d}(7.48 \mu \mathrm{m}$ for gel dominant, $8.69 \mu \mathrm{m}$ for solvent dominant environments). As a result, segregated aliquots employed for this work were generated in solvent dominant systems and segregated via gravity to afford maximum separation. More detailed information regarding microscopic analysis of samples can be found in Section S1.2.2 Microscopy.

To further improve the maximum $\Delta d$ between samples, the first round of segregated gel was segregated once more and decanted into five layers per aliquot. The relationship between singly segregated (parent) microsphere aliquots and doubly segregated (child) aliquots) is shown in Fig. S1. While iterative segregation achieves a higher degree of separation, solvent dominant environments necessarily produce a lesser amount of segregated gel product than gel dominant environments in identical volumes. To afford appropriate quantities of segregated gel for experimental analysis, samples resulting from the second round of segregation which exhibited similar mean bead surface area were combined. These combinations resulted in a total of four samples with unique mean diameter. Samples were microscopically analyzed using a Leica digital microscope and their diameters recorded with the aid of a Mathematica

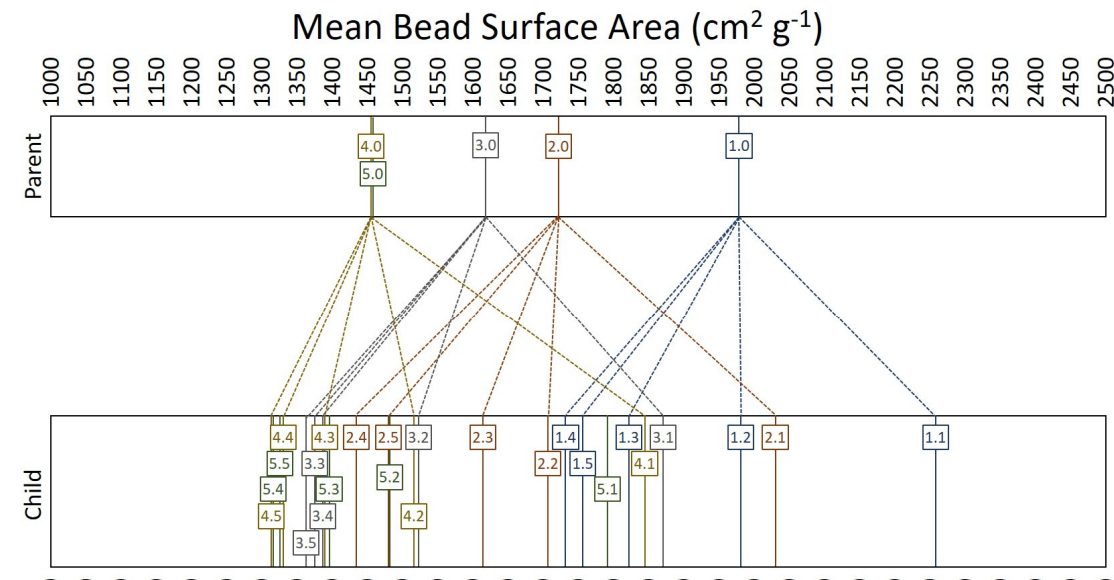

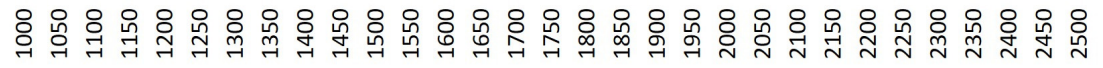

Fig. S1. Chart representing the size segregation of Sephacryl S200 microspheres via gravitational separation. Samples are labelled first with a number designating their respective parent sample (i.e. 1.1, meaning the sample is derived from parent one, taken from the top of the originally segregated sample of S200) followed by a numerical designation indicating which layer the sample was segregated from (i.e. 1.1, indicating the sample was removed from the top layer). Samples with roughly equivalent surface area per unit volume were combined to afford enough material for experimental analysis by column based SWNT purification.

program written to detect and size spherical objects.

\section{S1.2.2 Microscopy}

Microscopic analysis of samples was performed using a Leica digital microscope and capture software. All samples were homogenized by manual inversion prior to analysis to ensure the removal of a homogeneous volume for analysis. Sample analysis was performed by first loading a small amount $(\sim 0.10 \mathrm{~mL})$ of homogenized Sephacryl onto a glass microscope slide. The slide was then positioned beneath the microscope lens and fitted with a microscope cover glass to prevent evaporation of solvent (which results in beads physically shrinking) or significant movement of the beads caused by fluid motion. Images of bead samples were captured using a 10x objective and processed by a Mathematica program written to detect and size spherical objects in photographs. Diameters were calculated in terms of image pixels, then converted to micrometers with the aid of a microscope magnification standard slide. 


\section{S1.2.3 Determination of mass equivalence for hydrogel materials}

Because experimental procedures described here required changes in the aqueous volume of gel samples, it was necessary to determine the mass of gel in each sample for appropriate scaling of experimental quantities. This was done to provide surface area to material mass ratios for each sample and allow for the precise control of material quantities included in later experiments. A direct mass equivalence of segregated gel samples (obtained as described in Section S1.2.1) was afforded by placing equal volume amounts $(\sim 1.0 \mathrm{~mL})$ of each gel into a container under a rough pump generated vacuum for forty-eight hours to remove the aqueous layer. Comparison of these dried samples was made by taking the mass of each, and determination of the total gel mass made by accounting for a $2 \%$ by weight volume of aqueous Sodium Dodecyl Sulfate solution by subtraction of that mass.

\section{S1.2.4 Saturation of gel microsphere beds with SWNT}

In order to ensure the accuracy of quantitative spectroscopic analysis of SWNT suspensions passed through hydrogel beds of varied surface area it was necessary to allow for the analysis of such solutions without extensive sample dilution. Such quantitative analysis further required total saturation of each gel bed to neglect the effect of possible differences in SWNT uptake as a result of the lower SWNT concentration utilized. This was achieved by developing a procedure which utilized a relatively dilute solution of SWNT, which itself was compensated for by increased reaction time to allow for complete saturation of the gel beds. The general procedure for this experiment involved the loading of $31 \mathrm{mg}$ dried gel equivalent (see Supporting Information, Section S1.2.3) of each of presorted gels exhibiting 2157, 1826,1482 , and $1442 \mathrm{~cm}^{2} / \mathrm{g}$ to fritted columns with a $7 \mathrm{~mm}$ inner diameter (this mass was determined by scaling mass used in a typical SWNT separation experiment to the smaller column volume and gel bed diameter used here). ${ }^{1,2}$ This volume of gel, which was previously equilibrated in $70 \mathrm{mM}$ SDS solution, was then interacted with $2.18 \mathrm{~mL}$ of dilute SWNT solution. Interaction was maintained for a total of 60 minutes by iteratively adding this $2.18 \mathrm{~mL}$ suspension of SWNT to the top of the gel bed it passed through in a manner that avoided agitation of (and subsequent changes to) gel bed height following each pass through the gel bed. Following the conclusion of this extended interaction period SWNT suspensions (designated as post-gel-interaction) were collected and set aside for spectroscopic analysis. Gel beds were then washed with $2.0 \mathrm{~mL}$ of $70 \mathrm{mM}$ SDS solution to remove residual SWNT present in the inter-microsphere space but unbound to the gel, which was then discarded. Elution of the saturated gel beds was afforded by the subsequent addition of $1.0 \mathrm{~mL}$ of $175 \mathrm{mM}$ SDS solution, which was collected and used in the quantitative analysis of gel bed eluents and relative comparison of process efficiency for the four sorted gel samples used in this work.

\section{S1.2.5 Iterative purification of SWNT using column-based and centrifuge/decant-based methods}

The iterative purification of SWNT by chirality was performed using two methods. The standard column method and the novel centrifuge/decant method. For comparison, a SWNT purification using native Sephacryl S200 was run using both methods while mechanically fractured Sephacryl was only employed within the centrifuge/decant method due to its tendency to clog porous frits. The overall structure of the iterative purification involves using ten distinct gel beds and successively interacting the same SWNT solution with each. For the column method, $1.2 \mathrm{~mL}$ of the SWNT solution was placed into a column containing $21.25 \mathrm{mg}$ dried gel equivalent (see Supporting Information, Section S1.2.3) of native Sephacryl. To control the flow rate of SWNT through the column, a syringe pump was used and set to 
$0.12 \mathrm{~mL} / \mathrm{min}$ so that the SWNT/gel interaction would occur over 10 minutes (gravitational passing would occur over the course of $\sim 2$ minutes using these parameters). The SWNT solution was collected after interaction with the gel bed and retained for interaction with the following iteration of neat hydrogel. The column was then washed twice with $1.2 \mathrm{~mL}$ of $70 \mathrm{mM}$ SDS to remove residual SWNT present in the intermicrosphere space but unbound to the gel, which was then discarded. Finally, $1.2 \mathrm{~mL}$ of $175 \mathrm{mM}$ SDS were then passed through the gel bed to elute a fraction of the SWNT. This solution was saved for spectroscopic analysis. This procedure was repeated for each of the ten columns.

The centrifuge/decant method was performed in $2 \mathrm{~mL}$ Eppendorf centrifugation tubes. The same amount of Sephacryl as used in the column method, $21.25 \mathrm{mg}$ dried gel equivalent (see Supporting Information, Section S1.2.3), was placed in each tube. Again, $1.2 \mathrm{~mL}$ of post-ultracentrifugation SWNT solution was placed in the tube. The gel and SWNT solutions were homogenized on a vortex mixer, placed on the digital tube revolver, and rotated for 10 minutes. The tube was then transferred to the Sorvall MX 120 Plus Micro-Ultracentrifuge and centrifuged at maximum speed, $21 \mathrm{k} \mathrm{x} \mathrm{g}$, for 5 minutes. The SWNT solution was carefully decanted into a fritted column to prevent the transfer of gel. The filtered solution was collected and saved for the next iteration. The gel bed was washed twice with $1.2 \mathrm{~mL}$ of $70 \mathrm{mM}$ SDS by SDS addition, vortex mixing, and centrifugation ( $5 \mathrm{~min}, 21 \mathrm{kx} \mathrm{g}$ ) to afford phase separation. After each centrifugation the wash was carefully decanted into waste so as to retain the gel (and bound SWNT). Finally, $1.2 \mathrm{~mL}$ of $175 \mathrm{mM}$ SDS was added to the tube to elute the SWNT, vortex mixed, and placed on the revolver for 10 minutes. The tube was then centrifuged for 5 minutes. The solvent was carefully decanted into a fritted column to prevent any gel transfer. The solution was collected and saved for spectroscopic analysis. The purification with the mechanically fractured Sephacryl was performed in the same manner as the native Sephacryl except that only $2.125 \mathrm{mg}$ dried gel equivalent (see Supporting Information, Section S1.2.3) of gel was used per iterative SWNT/gel interaction. 


\section{S2. Spectroscopic deconvolution of SWNT absorbance spectra}

To gain quantitative insight from the collected absorbance spectra of SWNT in sodium dodecyl sulfate (SDS) aliquots, a deconvolution algorithm was developed and employed. This algorithm involved identifying the least squares best-fit in energy-space between a given experimental absorbance spectrum and a sum of simulated absorbance profiles of SWNT and an amorphous carbon background. Each SWNT chirality was assumed to exhibit a lowest-energy electronic transition $\left(E_{11}\right)$ along with a blue shifted and relatively lower intensity phonon sideband, both with Lorentzian line shape. The simulated absorbance spectrum was determined by:

$$
A_{\text {model }}(E)=\sum_{n, m} A_{n, m}(E)+A_{\mathrm{bkrd}}(E)
$$

where $A_{n, m}(E)$ is the chirality specific contribution to the total absorbance spectrum and $A_{\mathrm{bkrd}}(E)$ is the contribution from amorphous carbon background. Note that no contributions from the surfactant (SDS) are present in Eq. 1 because experimental spectra were collected against aqueous solutions of SDS concentration equal to that of the SWNT suspensions of interest.

The contribution of each SWNT chirality toward the observed absorbance was simulated using:

$$
A_{n, m}(E)=I_{n, m} \frac{\gamma_{\mathrm{SWNT}}^{2}}{\left(E-\left(E_{0}\right)_{n, m}\right)^{2}+\gamma_{\mathrm{SWNT}}^{2}}+f_{\mathrm{psb}} I_{n, m} \frac{\gamma_{\mathrm{psb}}^{2}}{\left(E-\left(E_{0}\right)_{n, m}+\left(E_{0}\right)_{\mathrm{psb}}\right)^{2}+\gamma_{\mathrm{psb}}^{2}}
$$

where $I_{n, m}$ is the chirality dependent height of the $\mathrm{E}_{11}$ absorbance band, $\gamma_{\mathrm{SWNT}}$ is the half-width at halfmaximum of the chirality specific $E_{11}$ absorbance band (held constant across all chiralities in the fit), $\left(E_{0}\right)_{n, m}$ is the energy-space center of the chirality specific $\mathrm{E}_{11}$ absorbance band, $f_{\mathrm{psb}}$ is the fraction of phonon side band height relative to that of the $E_{11}$ absorbance band (held constant across all chiralities in the fit), $\gamma_{\text {psb }}$ is the half-width at half-maximum of the phonon side band (held constant across all chiralities in the fit), and $\left(E_{0}\right)_{\mathrm{psb}}$ is the energy space blue shift of the phonon side band relative to the $E_{11}$ absorbance band (held constant across all chiralities in the fit).

The contribution of background features to absorbance spectra arise from a combination of amorphous carbon present from the prolonged tip horn sonication of as-synthesized SWNT to afford material suspension ${ }^{3}$ and Raleigh scattering from hydrogel fractions larger than optical wavelengths that were not isolated using experimental means. These contributions were simulated using:

$$
A_{\mathrm{bkrd}}(E)=I_{\mathrm{blk}} e^{-0.00155 * \frac{1241}{E}}+I_{\mathrm{N} 134} e^{-0.00300 * \frac{1241}{E}}+I_{\text {Rayleigh }}\left(\frac{1241}{E}\right)^{-4}
$$

where $I_{\mathrm{blk}}, I_{\mathrm{N} 134}$, and $I_{\text {Rayleigh }}$ are the intensity contributions of carbon black, Continental Carbon product $\mathrm{N} 134$, and Rayleigh scattering, respectively, the factor of 1241 represents a unit conversion from $\mathrm{nm}$ to $\mathrm{eV}$, 
and the exponential coefficients arise from a previous study of fitting background contributions to SWNT spectra. $^{3}$

Within a given fit, there exist two chirality specific parameters for each fit SWNT chirality: peak intensity and peak center, the former of which is unrestricted in its range and the latter of which is restricted to 12 meV within the initial guess value. There are also four "global" SWNTspecific parameters that

\begin{tabular}{|l|l|l|l|}
\hline Chirality & $\left(E_{0}\right)_{n, m}[\mathrm{eV}]$ & $\varepsilon_{n, m}\left[\mathrm{M}_{\text {carbon }}^{-1} \mathrm{~cm}^{-1}\right]$ & $\frac{\text { carbon atoms }}{\text { SWNT length }}\left[\# \mathrm{~nm}^{-1}\right]$ \\
\hline$(5,4)$ & 1.48 & 11,000 & 73.29 \\
\hline$(7,3)$ & 1.25 & 6,600 & 83.41 \\
\hline$(6,4)$ & 1.42 & 5,200 & 81.81 \\
\hline$(6,5)$ & 1.27 & 6,700 & 89.52 \\
\hline$(7,5)$ & 1.22 & 4,500 & 97.97 \\
\hline$(9,1)$ & 1.36 & 7,700 & 89.52 \\
\hline
\end{tabular}

Table S1. SWNT chirality specific parameters for each of six SWNT chiralities fit within this study. In conjunction with the best-fit parameters list within Table $\mathbf{S 2}$ and the assumption of uniform SWNT length of $L_{S W N T}=300 \mathrm{~nm}$, per-spectrum values of per-chirality peak height can be translated to total number of SWNT. are shared across all chiralities: SWNT peak width, ratio of SWNT $E_{11}$ to phonon side band peak, phonon side band peak width, and blueshift of phonon side band peak center relative to $E_{11}$ peak center. Additionally, there are three background specific parameters that are fit within each spectrum.

Chirality dependent best-fit values of $I_{n, m}$ associated with each spectrum were then converted to \# of SWNT using previously published values of chirality dependent SWNT absorbance cross section ${ }^{4}$ in conjunction with geometric solution of the chirality dependent number of carbon atoms per SWNT length ${ }^{5}$ and taking all SWNT to be of uniform length $\left(L_{S W N T}=300 \mathrm{~nm}\right)$ following prolonged tip horn sonication. ${ }^{2}$ Relevant chirality specific parameters for each of six SWNT chiralities fit within this study are shown in Table S1, while relevant "global" parameters resulting from the fits performed in this work are listed in Table S2.

Fitting of a differential spectra (the absorbance spectrum of a SWNT suspension prior to gel interaction minus that following gel interaction) with this model demonstrates that the spectroscopic character of materials

\begin{tabular}{|l|l|}
\hline parameter & \multicolumn{1}{|c|}{ value } \\
\hline$\gamma_{\mathrm{SWNT}}$ & 0.0300 \\
\hline$f_{\mathrm{psb}}$ & 0.108 \\
\hline$\gamma_{\mathrm{psb}}$ & 0.120 \\
\hline$\left(E_{0}\right)_{\mathrm{psb}}$ & 0.240 \\
\hline
\end{tabular}

Table S2. Global (shared across chiralities) SWNT best-fit parameters used within this study. In conjunction with the chirality specific parameters list within Table S1 and the assumption of uniform SWNT length of $L_{S W N T}=300 \mathrm{~nm}$, perspectrum values of per-chirality peak height can be translated to total number of SWNT. adsorbed to the gel is SWNT in nature. This is shown for the gel bed exhibiting $56.9 \mathrm{~cm}^{2}$ of surface area in Fig. $2 \mathrm{C}$ within the main text. Here, the best-fits
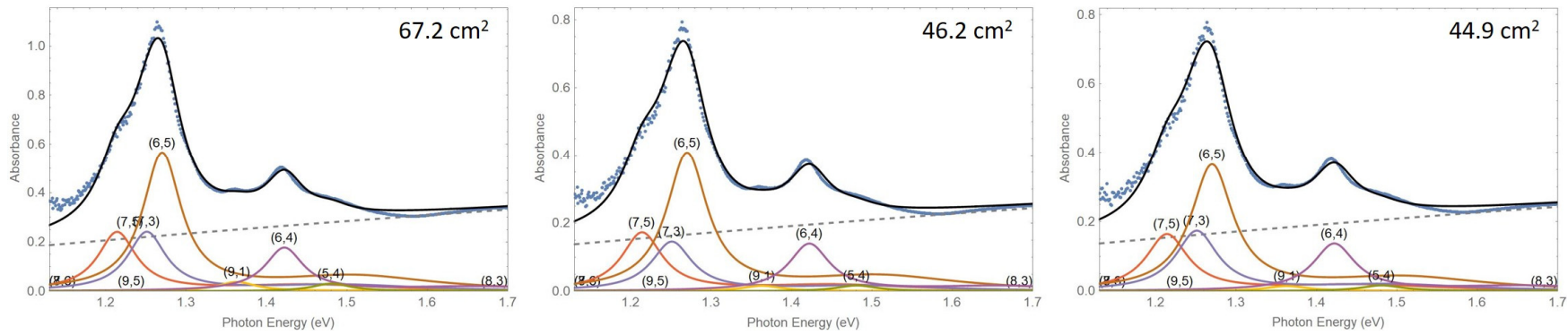

Fig. S2. Fitted differential absorbance spectra of SWNT suspensions before and after interacting with hydrogel beds exhibiting surface area of $67.2 \mathrm{~cm}^{2}, 46.2 \mathrm{~cm}^{2}$, and $44.9 \mathrm{~cm}^{2}$. Experimental spectra (black) are modeled by the sum of six Lorentzian line-shapes (multicolor) with constant FWHM of $60 \mathrm{meV}$ and corresponding phonon side band blue shifted by $0.240 \mathrm{eV}$ and of height $10.8 \%$ of that of the corresponding $\mathrm{E}_{11}$ peak. 
obtained for the other three gel beds exhibiting surfaces areas $67.2 \mathrm{~cm}^{2}, 46.2 \mathrm{~cm}^{2}$, and $44.9 \mathrm{~cm}^{2}$ are shown in Fig. S2. Additionally, the best-fit of the pre-gel-interaction SWNT suspension absorbance spectrum is shown in Fig. S3, which is represented as the black trace within Fig. 2 A. Chirality dependent quantitative results from each of the four fitted differential spectra (along with the pre-gel-interaction spectrum) are summarized in Table 1 within the main text.

Finally, the best-fit of the SWNT desorbed from each of the four gel beds exhibiting $67.2 \mathrm{~cm}^{2}$, $46.2 \mathrm{~cm}^{2}, 56.9 \mathrm{~cm}^{2}$, and $44.9 \mathrm{~cm}^{2}$, respectively, are shown in Fig. S4. These best-fits correspond with the four spectra shown in Fig. 4A of the main text, while chirality dependent quantitative results from each of the four fitted differential spectra are summarized in Table $\mathbf{2}$ within the main text.

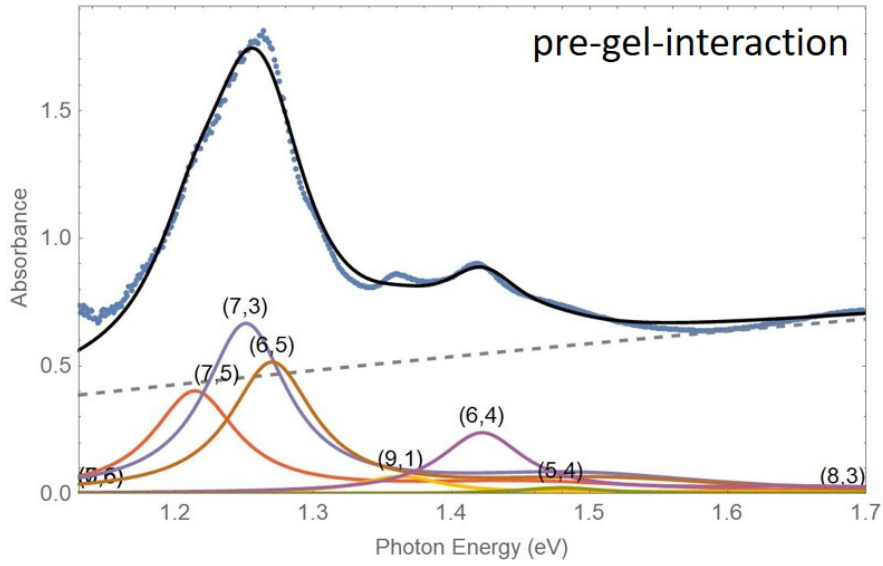

Fig. S3. Fitted absorbance spectrum of SWNT suspension prior to interaction with hydrogel microsphere beds exhibiting surface areas of $67.2 \mathrm{~cm}^{2}, 46.2 \mathrm{~cm}^{2}, 56.9 \mathrm{~cm}^{2}$, and $44.9 \mathrm{~cm}^{2}$.
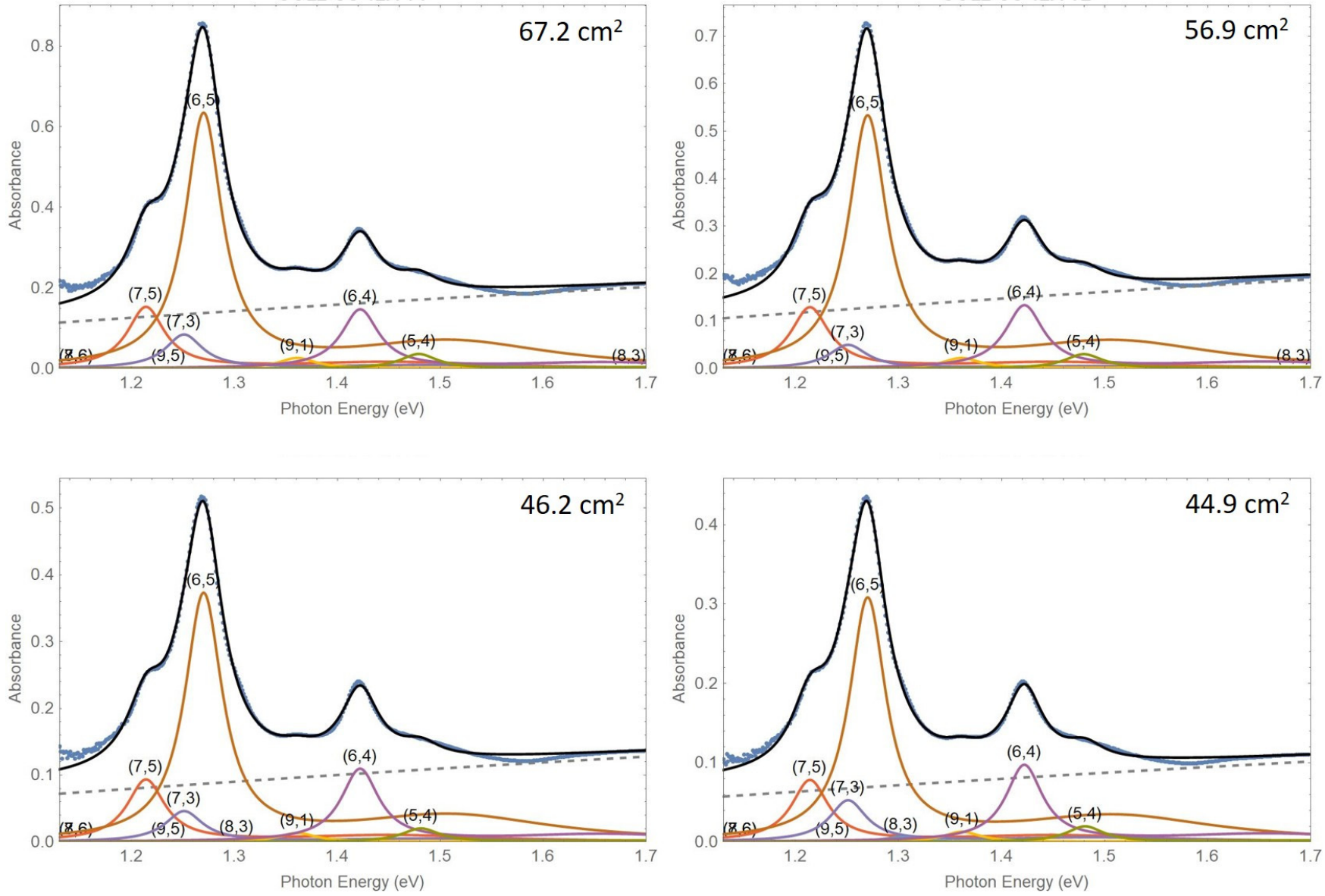

Fig. S4. Fitted absorbance spectra of SWNT suspensions desorbed from hydrogel beds exhibiting surface area of $67.2 \mathrm{~cm}^{2}, 46.2 \mathrm{~cm}{ }^{2}, 56.9$ $\mathrm{cm}^{2}$, and $44.9 \mathrm{~cm}^{2}$ following introduction of $175 \mathrm{mM}$ SDS. 


\section{S3. Geometric model of SWNT interaction with curved surface of hydrogel microsphere}

A geometric relationship between a curved microsphere surface and a rigid SWNT of length LsWNT with midpoint placed tangentially to the surface of the sphere affords insight into the fraction of LsWNT $_{\text {that }}$ exists at distances whereby SWNT/gel interaction is energetically favorable. These distances are assumed to occur within the SWNT/gel separation distance range of $D_{\min }-D_{\max }$. A schematic to aid in the visualization of the derivation portion of this model is shown in Fig. S5. First, one can consider a rigid rod of length LsWNT with midpoint positioned at an angle normal to the surface of a sphere with radius $R$ and separated from that surface by distance $D_{\text {min. }}$. This geometric setup results in a minimum SWNT/gel separation distance of $D_{\min }$ and the remainder of the SWNT length exhibiting separation distance that may be less than or greater than $D_{\max }$. Consideration of a point on the surface of the sphere removed from the line normal to the SWNT surface by angle $\theta$ affords consideration of the corresponding point of shortest distance on the SWNT surface, so long as the longitudinal distance traveled is

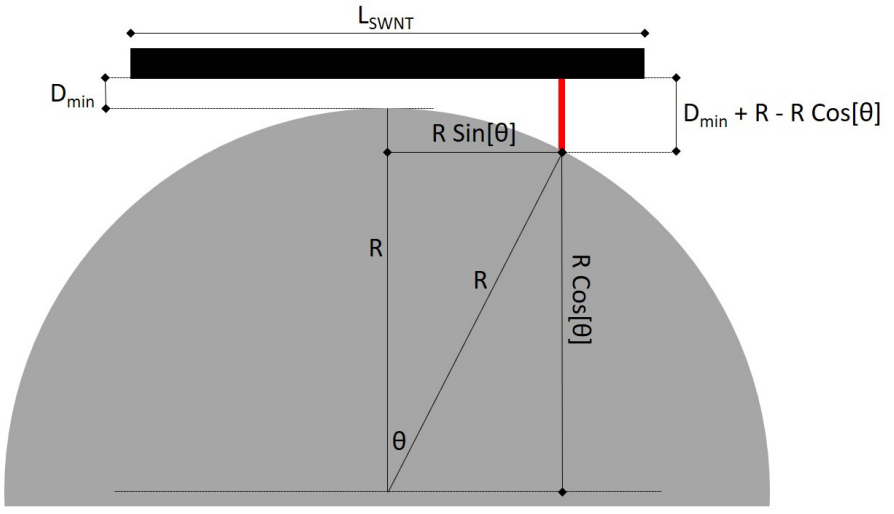

Fig. S5. Schematic diagram outlining relevant spatial parameters in the derivation of the fraction of SWNT length exhibiting spatial separation from a curved surface within separation distance $D_{\min }-D_{\max }$.

$$
R \operatorname{Sin}(\theta)<\frac{L_{S W N T}}{2}
$$

So long as the condition in Eq. 4 is met, the shortest separation distance between that point and any point on the surface of the SWNT is given by:

$$
D_{S W N T / g e l}=D_{\min }+R-R \operatorname{Cos}(\theta)
$$

as indicated by the red vertical line within Fig. S5. This model then assumes that a hard cutoff (separation distance) exists whereby the SWNT and gel surfaces no longer interact in an energetically favorable way, which is defined as $D_{\text {max }}$. To identify the fraction of the SWNT length lying within the spatial range of $D_{\min } \leq D_{s W N T / g e l}$ $\leq D_{\max }$, the point on the gel surface whereby $D_{\text {sWNT/gel }}=D_{\max }$ becomes of critical interest. This point corresponds with a critical angle $\theta_{\text {crit, }}$ as defined by:

$$
D_{\max }=D_{S W N T / g e l}=D_{\min }+R-R \operatorname{Cos}\left(\theta_{\text {crit }}\right)
$$

Solving for the critical angle in terms of system constants yields:

$$
\theta_{\text {crit }}=\operatorname{ArcCos}\left(\frac{R+D_{\min }-D_{\max }}{R}\right) .
$$

The position along the length of the SWNT removed from its halfway point corresponding to $\theta_{\text {crit }}$ is: 


$$
P_{S W N T}=R \operatorname{Sin}\left(\theta_{c r i t}\right)=R \operatorname{Sin}\left(\operatorname{ArcCos}\left(\frac{R+D_{\min }-D_{\max }}{R}\right)\right)
$$

which holds so long as the relationship in Eq. 4 with $\theta=\theta_{\text {crit }}$ is met, which, in that case, the total fraction of the SWNT length with surface laying within the spatial range of $D_{\min } \leq D_{S W N T / g e l} \leq D_{\max }$ is given by (due to the symmetry of the system):

$$
f_{L}=\frac{P_{S W N T}}{L / 2}=\frac{2 R}{L} \operatorname{Sin}\left(\operatorname{ArcCos}\left(\frac{R+D_{\min }-D_{\max }}{R}\right)\right) .
$$

If, however, the relationship in Eq. 4 with $\theta=\theta_{\text {crit }}$ is not met this means that the point on the gel surface at which $D_{\text {SWNT/gel }}=D_{\max }$ is beyond the end of the SWNT. In other words, the entirety of the SWNT length lays within the spatial range of $D_{\min } \leq D_{\text {sWNT/gel }} \leq D_{\max }$, and definitionally:

$$
f_{L}=1
$$

Substituting the expression for $\theta_{\text {crit }}$ from Eq. 7 into the condition expressed in Eq. 4 yields a condition for the utilization of Eq. 9 vs. Eq. 10 in terms of system parameters:

$$
2 R \operatorname{Sin}\left(\operatorname{ArcC} \operatorname{os}\left(\frac{R+D_{\min }-D_{\max }}{R}\right)\right)<L_{S W N T} .
$$

Given the trigonometric definition of $\operatorname{Sin}(\operatorname{Arc} C \operatorname{Oos}(x))=\sqrt{1-x^{2}}$, Eqs. 9, 10, and 11 can be combined to yield a simplified conditional expression for $\mathrm{f}_{\mathrm{L}}$ :

$$
f_{L}=\left\{\begin{array}{cl}
\frac{2 R}{L} \sqrt{1-\frac{\left(R+D_{\min }-D_{\max }\right)^{2}}{R^{2}}} & \text { if } 2 R \sqrt{1-\frac{\left(R+D_{\min }-D_{\max }\right)^{2}}{R^{2}}}<L_{S W N T} \\
1 & \text { if } 2 R \sqrt{1-\frac{\left(R+D_{\min }-D_{\max }\right)^{2}}{R^{2}}} \geq L_{S W N T}
\end{array}\right\} .
$$

A plot of Eq. 12 for the parameters utilized in this study $\left(D_{\min }=0.127 \mathrm{~nm}\right.$ and $\left.D_{\max }=0.313 \mathrm{~nm}\right)$ is shown in Fig. S6. Note that for these conditions and with an assumed $L_{S W N T}=300 \mathrm{~nm}$, all hydrogel microspheres with $\mathrm{R}>$ $60.48 \mu \mathrm{m}$ exhibit sufficiently minimal curvature relative to LsWNT that the entirety of the SWNT length lays within the spatial range of $D_{\min } \leq D_{\text {swnT/gel }} \leq D_{\max }$. While this critical hydrogel microsphere size may be of interest for future studies, all microspheres observed within this work had $R \leq 37 \mu \mathrm{m}$, indicating that all SWNT/gel interactions in this work involved $\mathrm{f}_{\mathrm{L}}<1$.

Discussion of Model Assumptions. While Eq. 12 affords relatively straightforward insight into what may be a critical parameter driving the reversibility of SWNT/gel interactions, it is also important to note the assumptions and limitations of this model. Such assumptions can be lumped into one of two categories: geometric and energetic. First, two geometric assumptions are made that detract from the physical accuracy 
of this model. The first geometric assumption is that an inherently three-dimensional system can be captured with a two-dimensional description, or in other words, that the curvature of the SWNT surface can be neglected in describing its interaction with a gel microsphere. The second geometric assumption is the pristine nature of both the SWNT and the gel microspheres, that is, that the SWNT is perfectly straight and the gel microsphere is an otherwise flat surface. Additionally, this model makes two assumptions that are energetic in nature. The first energetic assumption is that there exists a hard distance cutoff whereby a SWNT/gel system does (or does not) interact in an energetically/thermodynamically favorable way. The second energetic assumption is that the distance over which energetically favorable SWNT/gel interactions, $D_{\min } \leq D_{\text {sWNT/gel }} \leq D_{\max }$, is independent of SWNT chirality.

In terms of the geometric assumptions made, this

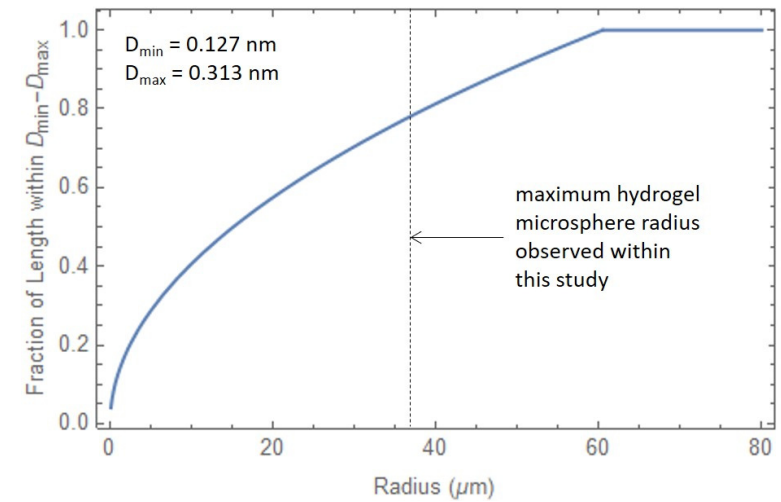

Fig. S6. Relationship between hydrogel microsphere radius and $\mathrm{fL}$ for the geometric parameters used within this study, $D_{\min }=0.127 \mathrm{~nm}, D_{\max }=0.313 \mathrm{~nm}$, and $L_{S W N T}=300 \mathrm{~nm}$. While any hydrogel microsphere with $\mathrm{R}>60.48 \mu \mathrm{m}$ is predicted to exhibit sufficiently minimal curvature relative to $L_{S W N T}$ that the entirety of the SWNT length lays within the spatial range of $D_{\min } \leq$ DSWNT/gel $\leq D_{\max }$, the largest Sephacryl microsphere radius observed in this study was $37 \mu \mathrm{m}$. model strikes a strong balance between complexity and expected accuracy. While neglecting SWNT curvature by treating the system as two-dimensional does not account for potential SWNT/gel interactions occurring at distances slightly greater than the minimum SWNT/gel separation at a given point on the gel surface, those interactions are expected to generally track in accord to those accounted for within this model. Further, the assumption that the hydrogel surface is geometrically uniform is necessary for this model (in terms of arriving at a straightforward expression), albeit inaccurate. Specifically, Sephacryl is manufactured to exhibit pore diameter of $\sim 10 \mathrm{~nm}$ to afford its use as size exclusion chromatography media, meaning that spatial and chemical inhomogeneities on the tens-ofnanometers level are expected. However, given that the SWNT interacting with the gel surface has length on a hundreds-of-nanometers scale, gel surface inhomogeneities likely have a relatively minimal effect on the geometric nature of SWNT/gel distance. On a related note, it is expected that the Sephacryl gel itself may exhibit chemical regions more-dense in some monomers/polymer configurations than others. To better account for this, a sigmoid function was used in the main text (as opposed to a hard cutoff) when considering what $f_{L}$ afford reversibility of the SWNT/gel binding event.

In terms of the energetic assumptions made, this model also strikes a balance between complexity and expected accuracy. While system energy vs. separation distance would be most accurately characterized by a continuum, using a hard-cutoff affords capturing system behavior within a single parameter: fraction of SWNT length, which has a straightforward physical interpretation and provides straightforward experimental predictions for future studies. Additionally, the assumption that all SWNT chiralities exhibit the same energetic profile against gel separation distance contradicts the chirality-dependent nature of SWNT/gel interactions that makes them so enticing within SWNT purification schemes. To better tease out these effects, one would need to repeat experimentation introduced in this study but with single-chirality samples of SWNT. Perhaps ironically, preparative quantities of single chirality SWNT are only possible for a few SWNT chiralities, and access to the materials necessary to conduct such a study would first require further research into the nature of the SWNT/gel interaction to better understand and improve upon this method for purification.

Overall, the model developed in this work to afford insight into the desorption efficiency of SWNT/gel binding is useful in both its relative simplicity and its relative accuracy. Its benefits include the identification of the process as thermodynamically driven, its identification of a straightforward geometric parameter that drives the process, and its suggestion of future directions to improve process efficiency. 


\section{S.4 Comparison between results obtained using column-based and centrifuge-based methods to achieve gel/SWNT phase separation within SWNT purification scheme}

As discussed in the main text, the typical "column" configuration (use of a frit to achieve phase separation between the hydrogel and the SWNT suspension) was not possible when performing SWNT/gel interactions with the mechanically fractured Sephacryl. Because the mechanically fractured Sephacryl had hydrogel bits at-or-below the average pore size of the frit, this material would cause the frit to clog and prevent flow of the SWNT suspension. To circumvent this issue, a batch reactor configuration was utilized that relied on centrifugation/decanting to achieve SWNT/gel separation instead of a frit. To demonstrate the equivalence between a standard "fritted column" based scheme and a "centrifuge/decant" based scheme, we present both qualitative photographs and quantitative spectra.

First, images of the per-iteration SWNT aliquots released from gels under three different configurations-fritted column configuration using native Sephacryl S200 at $21 \mathrm{mg}$ dried gel per iteration, centrifuge/decant configuration using native Sephacryl S200 at $21 \mathrm{mg}$ dried gel per iteration, and centrifuge decant configuration using mechanically fractured Sephacryl at $2.1 \mathrm{mg}$ dried gel per iteration-are shown in Fig. S7. The coloration of the eluants from these procedures both demonstrates that a tenfold smaller amount of gel (exhibiting larger surface area) can be used to achieve nearly identical SWNT

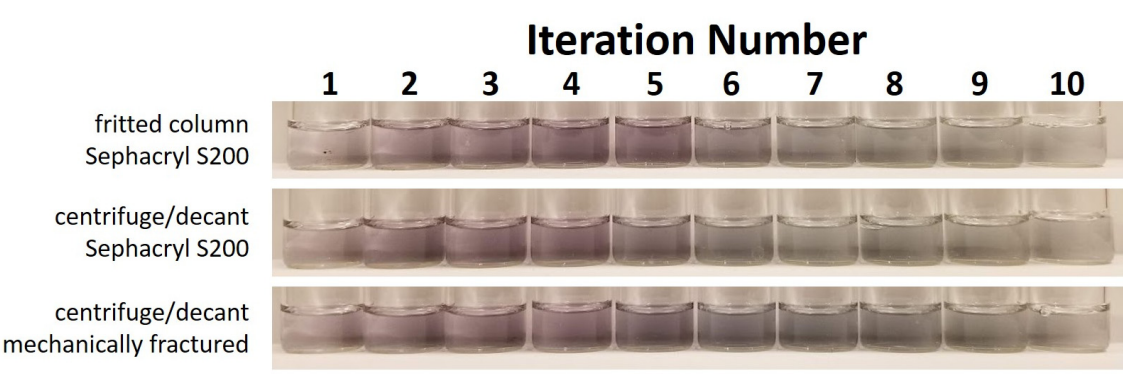

Fig. S7. Visual comparison of fritted column based and centrifuge/decant based methods. Samples shown are eluent acquired from separating the gel and surrounding liquid phase by (top) passing the SWNT suspension through a fritted column to prevent the passage of hydrogel microspheres and (bottom two) centrifugation of the SWNT-gel matrix followed by careful decanting. Qualitative comparisons displayed here are accompanied by spectroscopic analysis of each sample to afford evidence supporting the use of lower masses of fractionated hydrogel for SWNT purification.

Fritted Column Sephacryl S200

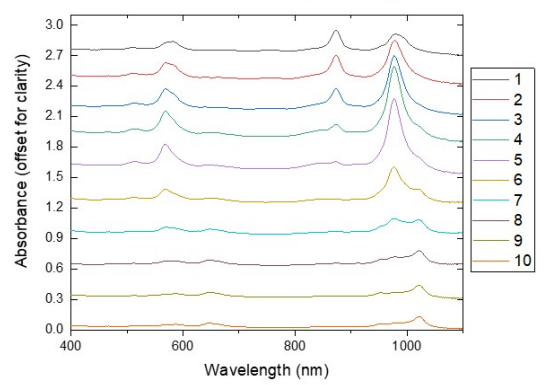

Fritted Column Sephacryl S200

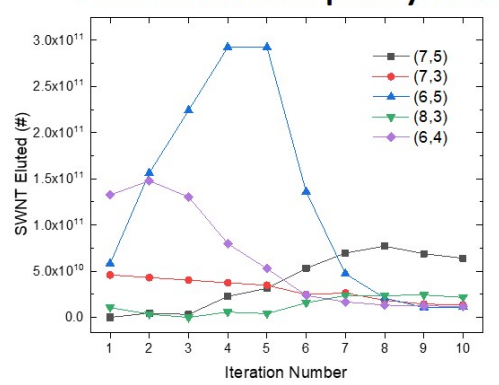

Fig. S8. Side-by-side comparison showcasing the ability of both a fritted column configuration and a centrifuge/decant configuration to achieve similar SWNT purification results within an iterative gel-based scheme. The similarity quantified here demonstrates that both a centrifuge/decant method is appropriate for demonstrating the viability of a reduced hydrogel scheme (with higher gel surface area via mechanical fractionation) and the general viability of scaled purification of SWNT by chirality using a centrifuge/decant approach. 
purification (similar finding to that showcase in the main text), as well as that a fritted column based purification scheme also yields nearly identical results in comparison with a centrifuge/decant based purification scheme. In the case of each of the three images, it is clear that chirally purified SWNT result from the applied techniques and that the SWNT chirality makeup changes over the course of a ten-iteration procedure.

Second, to quantitatively compare the similarity between fritted column and centrifuge/decant based SWNT/gel interaction methods, absorbance spectra and the resultant deconvoluted per-chirality best-fit of those spectra are compared side-by-side for the two techniques over a 10-iteration purification in Fig. S8. This analysis provides further evidence that the centrifuge/decant method is a viable alternative to the traditional fritted column method. Further, general scalability of a centrifuge/decant configuration makes this approach enticing for large-scale purification of SWNT by chirality. 


\section{References}

(1) Liu, H.; Nishide, D.; Tanaka, T.; Kataura, H. Large-Scale Single-Chirality Separation of Single-Wall Carbon Nanotubes by Simple Gel Chromatography. Nature Communications 2011, 2, 309.

(2) Tvrdy, K.; Jain, R. M.; Han, R.; Hilmer, A. J.; McNicholas, T. P.; Strano, M. S. A Kinetic Model for the Deterministic Prediction of Gel-Based Single-Chirality Single-Walled Carbon Nanotube Separation. ACS Nano 2013, 7 (2), 1779-1789.

(3) Naumov, A. V.; Ghosh, S.; Tsyboulski, D. A.; Bachilo, S. M.; Weisman, R. B. Analyzing Absorption Backgrounds in Single-Walled Carbon Nanotube Spectra. ACS Nano 2011, 5 (3), 1639-1648.

(4) Sanchez, S. R.; Bachilo, S. M.; Kadria-Vili, Y.; Lin, C.-W.; Weisman, R. B. ( $N$, $m$ )-Specific Absorption Cross Sections of Single-Walled Carbon Nanotubes Measured by Variance Spectroscopy. Nano Letters 2016, 16 (11), 6903-6909.

(5) Reich, S.; Thomsen, C.; Maultzsch, J. Carbon Nanotubes: Basic Concepts and Physical Properties, 2. Reprint.; Wiley-VCH: Weinheim, 2005. 